\title{
Exact dynamical response of an N-electron quantum dot subject to a time-dependent potential
}

\author{
Simon C. Benjamin and Neil F. Johnson \\ Physics Department, Clarendon Laboratory, Oxford University, Oxford OX1 3PU, England
}

\begin{abstract}
We calculate analytically the exact dynamical response of a droplet of $N$ interacting electrons in a quantum dot with an arbitrarily time-dependent parabolic confinement potential $\omega(t)$ and a perpendicular magnetic field. We find that, for certain frequency ranges, a sinusoidal perturbation acts like an attractive effective interaction between electrons. In the absence of a timeaveraged confinement potential, the $N$ electrons can bind together to form a stable, free-standing droplet.
\end{abstract}

PACS numbers: 73.61.-r, 03.65.Ge, 73.23.-b 
Quantum dots have attracted much interest recently, both from a pure and applied viewpoint. According to the dot fabrication, the confinement length scales in the three spatial directions can be quite different yielding quasi one-, two- or three-dimensional dots 11. In addition the number of electrons $N$ in the dot can be reduced down to the few-electron limit. Most theoretical quantum dot research has been concerned with the linear response of the resulting $N$-electron, low-dimensional system. External, time-dependent electric fields are typically treated as small perturbations which merely give rise to transitions between the eigenstates of the unperturbed dot. Given the technological possibilities for preparing stronger fields, it is interesting to consider the effects of larger, time-dependent perturbations on such dots. For example, a confinement potential with sinusoidal time-dependence could be created by applying an a.c. bias to the electrodes defining the dot in a heterostructure sample. Unfortunately, the quantum-mechanical problem of $N$ interacting electrons in a dot subject to an arbitrarily strong, time-varying perturbation is too complicated to solve in general, even numerically.

Here we provide an analytically solvable model for the dynamical response of an $N$ electron dot with an arbitrarily strong, time-varying confinement potential, in the presence of an arbitrarily strong magnetic field $B$. The analytic tractability of the model is made possible through a combination of a parabolic form for the dot confinement potential and an inverse-square electron-electron interaction potential $\left(1 / r^{n}\right.$ with $\left.n=2\right)$. The parabolic confinement is a reasonable approximation for many semiconductor quantum dot samples [1]. The true repulsive interaction between electrons in the dot is likely to be better fit by $n \sim 1$ at small $r$ and $n \sim 3$ at large $r$ due to image charge effects in neighboring electrodes [2]; however the general features of our results with $n=2$ for all $r$ should be qualitatively correct. Here we focus on the usual quasi-two-dimensional dot; however, most of our formal results can be generalized to both quasi-one- and quasi-three-dimensional dots at $B=0$.

The time-dependent Schrodinger equation for the dot with a time-dependent confinement in the xy-plane, subject to a constant $B$ field applied along z, is given within the effective- 
mass approximation by $H(t) \Psi(t)=i \hbar \frac{\partial}{\partial t} \Psi(t)$ with

$$
H(t)=\sum_{i}\left(\frac{\mathbf{p}_{i}^{2}}{2 m^{*}}+\frac{1}{2} m^{*} \omega^{2}(t)\left|\mathbf{r}_{i}\right|^{2}+\frac{\omega_{c}}{2} l_{i}\right)+\sum_{i<j} \frac{\xi}{\left|\mathbf{r}_{i}-\mathbf{r}_{j}\right|^{2}} .
$$

The momentum, position and z-component of angular momentum of the $i$ 'th electron are given by $\mathbf{p}_{i}, \mathbf{r}_{i}$ and $l_{i}$. The cyclotron frequency is $\omega_{c}$ (N.B. we can only solve with $\omega_{c} \neq 0$ for a two-dimensional dot). The spin part of the Hamiltonian is time-independent and therefore decouples for all $t$. In order to include both transient and steady-state responses, we consider the time-dependent dot potential to be turned on at time $t=0$ :

$$
\omega^{2}(t)= \begin{cases}\omega_{0}^{2}(B)=\omega_{0}^{2}+\omega_{c}^{2} / 4 & \text { for } t \leq 0 \\ f(t) & \text { for } t>0\end{cases}
$$

where $\omega_{0}$ is the characteristic frequency of the parabolic confinement potential for $t \leq 0$, and $f(t)$ has arbitrary functional form and magnitude.

For $t<0, \omega^{2}(t)$ is time-independent. The problem is treated in Ref. [3]; here we review the essential results. Standard Jacobi coordinates are employed, $\mathbf{X}_{i}(i=0,1, \ldots, N-1)$ where $\mathbf{X}_{0}=\frac{1}{N} \sum_{j} \mathbf{r}_{j}$ (center-of-mass), $\mathbf{X}_{1}=\sqrt{\frac{1}{2}}\left(\mathbf{r}_{2}-\mathbf{r}_{1}\right), \mathbf{X}_{2}=\sqrt{\frac{2}{3}}\left(\frac{\left(\mathbf{r}_{1}+\mathbf{r}_{2}\right)}{2}-\mathbf{r}_{3}\right)$ etc. together with their conjugate momenta $\mathbf{P}_{i}$. The center-of-mass motion decouples, $H=H_{\mathrm{CM}}\left(\mathbf{X}_{0}\right)+$ $H_{\text {rel }}\left(\left\{\mathbf{X}_{i>0}\right\}\right)$, hence $E=E_{\mathrm{CM}}+E_{\text {rel }}$ and $\Psi=\psi_{\mathrm{CM}} \psi_{\text {rel }}$. The exact eigenstates $\psi_{\mathrm{CM}}$ of $H_{\mathrm{CM}}$ and eigenenergies $E_{\mathrm{CM}}$ are identical to those of a single particle in a parabolic potential. The non-trivial problem is to solve the relative motion equation $H_{\text {rel }} \psi_{\text {rel }}=E_{\text {rel }} \psi_{\text {rel }}$. We transform the relative coordinates $\left\{\mathbf{X}_{i>0}\right\}$ to standard hyperspherical coordinates: $\mathbf{X}_{i}=$ $r\left(\prod_{j=i}^{N-2} \cos \alpha_{j+1}\right) \sin \alpha_{i} e^{i \theta_{i}}$ with $r \geq 0$ and $0 \leq \alpha_{i} \leq \frac{\pi}{2}\left(\alpha_{1}=\frac{\pi}{2}\right)$. Physically, the hyperradius $r$ is just the root-mean-square electron-electron separation. The exact eigenstates of $H_{\text {rel }}$ have the form $\psi_{\text {rel }}=R(r) F(\tilde{\Omega})$ where $\tilde{\Omega}$ denotes the $(2 N-3)$ hyperangular $\{\theta, \alpha\}$ variables; $R(r)$ and $F(\tilde{\Omega})$ are solutions of the hyperradial and hyperangular equations respectively.

For $t>0$, the separation of the relative motion is still exact, i.e. $\Psi=\psi_{\mathrm{CM}}(t) \psi_{\text {rel }}(t)$. The hyperangular equation is independent of $\omega(t)$, hence $F(\tilde{\Omega})$ remains time-independent. The time-dependence of $\Psi$ is only contained in the center-of-mass and hyperradial parts. Consider explicitly $\psi_{\text {rel }}(t)$ and hence the hyperradial part $R(r, t)$; the solution for $\psi_{\mathrm{CM}}(t)$ is exactly analogous. Following Ref. [5] we construct the generating function 


$$
g(z, r, t) \equiv \sum_{n=0}^{\infty} R_{n}(r, t) z^{n}
$$

where $R_{n}(r, t)$ are the solutions of the time-dependent hyperradial equation. Because the dot potential is constant for $t \leq 0$, we can employ the static $R_{n}(r)$ from Ref. [3] to obtain

$$
g(z, r, t \leq 0)=\sum_{n=0}^{\infty}\left(\frac{r}{l_{0}}\right)^{\gamma} L_{n}^{\gamma+N-2}\left(\frac{r^{2}}{l_{0}^{2}}\right) e^{-\frac{r^{2}}{2 l_{0}^{2}}} z^{n}
$$

where $L$ denotes the Laguerre polynomial and $l_{0}^{2}=\hbar\left(m^{*} \omega_{0}(B)\right)^{-1}$. The parameter $\gamma$ is determined by the $\omega_{0}, B$ and $t$-independent hyperangular equation, which does not admit complete exact solution. Fortunately, we do not need any further knowledge of the properties of $\gamma$ for the analysis in this paper. Equation (4) can be written in closed form using a known identity [4]

$$
g(z, r, t \leq 0)=\left(\frac{r}{l_{0}}\right)^{\gamma} e^{\frac{z+1}{2(z-1)}\left(\frac{r}{l_{0}}\right)^{2}}(1-z)^{-(a+1)}
$$

where $a=\gamma+N-2$. We make the ansatz

$$
g(z, r, t>0)=\alpha(z, t)\left(\frac{r}{l_{0}}\right)^{\gamma} e^{\alpha^{\prime}(z, t) r^{2}}
$$

which can be shown to satisfy the time-dependent hyperradial equation

$$
\left(\frac{\partial^{2}}{\partial r^{2}}+\frac{2 N-3}{r} \frac{\partial}{\partial r}-\frac{\gamma(\gamma+2 N-4)}{r^{2}}-\left(\frac{m^{*}}{\hbar}\right)^{2} \omega^{2}(t) r^{2}\right) g(z, r, t)=-\frac{2 i m^{*}}{\hbar} \frac{\partial}{\partial t} g(z, r, t)
$$

and the $t=0$ boundary condition in Eq. (5), provided

$$
\alpha(z, t)=[\eta(t)]^{-(a+1)} \exp [2 i \theta(t)(a+1)](1-z \exp [2 i \theta(t)])^{-(a+1)}
$$

and

$$
\alpha^{\prime}(z, t)=\frac{i m^{*}}{2 \hbar}\left(\frac{\dot{\eta(t)}}{\eta(t)}-2 i \dot{i(t)}(1-z \exp [2 i \theta(t)])^{-1}\right)
$$

where $\eta(t)=|\eta(t)| e^{i \theta(t)}$ solves the classical one-dimensional oscillator

$$
\ddot{\eta(t)}+f(t) \eta(t)=0
$$


with boundary conditions $\eta(0)=1$ and $\dot{\eta(0)}=-i \omega_{0}(B)$. We may then expand $g(z, r, t)$ using the relevant identity [4], and compare coefficients of $z^{n}$ with the defining Eq. (3) to obtain the desired time-dependent wavefunctions (unnormalized):

$$
R_{n}(r, t>0)=|\eta(t)|^{1-N} y^{\gamma} \exp \left[i\left(\theta(t)(2 n+a+1)+\frac{y^{2}}{4 \omega_{0}(B)} \frac{d}{d t}|\eta(t)|^{2}\right)\right] e^{-\frac{1}{2} y^{2}} L_{n}^{a}\left(y^{2}\right)
$$

where $y \equiv \frac{r}{|\eta| l_{0}}$. Solving Eq. (10) hence provides a complete description for the evolution of the initially stationary hyperradial state $R_{n}$ for $t>0$. Equation (11) together with the exactly analogous expression for the center-of-mass entirely determines the time-evolution of the total wavefunction $\Psi$.

First we consider a step-like perturbation [6] (see Fig. 1(a))

$$
\omega^{2}(t)=\left\{\begin{array}{ll}
\omega_{0}^{2} & \text { for } t \leq 0 \\
f(t)=\omega_{\infty}^{2}-\frac{w_{\infty}^{2}-\omega_{0}^{2}}{(\Omega t+1)^{2}} & \text { for } t>0
\end{array} .\right.
$$

Increasing $\Omega$ reduces the time period over which the change occurs. The solution to Eq. (10) for $t>0$ is

$$
\eta(t)=(\Omega t+1)^{\frac{1}{2}}\left\{A J_{v}\left[\omega_{\infty}\left(t+\Omega^{-1}\right)\right]+B N_{v}\left[\omega_{\infty}\left(t+\Omega^{-1}\right)\right]\right\}
$$

where $J_{v}$ and $N_{v}$ are Bessel functions of the first and second kind respectively, $v=\left(\frac{\left(\omega_{\infty}^{2}-\omega_{0}^{2}\right)}{\Omega^{2}}+\right.$ $\left.\frac{1}{4}\right)^{\frac{1}{2}}$, and $A$ and $B$ are complex constants chosen to satisfy $\eta(0)=1$ and $\dot{\eta(0)}=-i \omega_{0}$. This complex function is displayed in Fig. 1(b), the inset on the right showing the view when projected on the complex $\eta$ plane. For $t<0$ the value of $\eta(t)$ lies on the unit circle in the complex plane, and describes a simple helix around the time axis as $t$ varies. For $t>0$ the function describes an ellipsoidal path in the complex $\eta$ plane, the ellipsoid lying entirely within the unit circle; the eccentricity of the ellipsoid is determined by the sharpness of the transition from $\omega_{0}$ to $\omega_{\infty}$, i.e. by $\Omega$. Since $R_{n}(r, t)$ (Eq. 11) depends on $y \equiv \frac{r}{|\eta| l_{0}}$, the ellipsoidal path of $\eta(t)$ implies that the total wavefunction will oscillate for all $t>0$. Figure 1(c) shows the time-dependence of the expectation value of the hyperradius $\bar{r}$ (r.m.s. electron-electron separation). Remarkably, this quantity oscillates for all $t>0$ regardless of the values of $\omega_{0}, \omega_{\infty}$ and $\Omega$. Changing $\Omega$ alters the oscillation amplitude but not the period. 
In order to obtain a large amplitude of oscillation, $\Omega^{-1}$ must be of comparable order to $\omega_{0}^{-1}$; if $\hbar \omega_{0} \sim 1 \mathrm{meV}, \Omega^{-1}$ should be of the order of picoseconds.

Second we consider a sinusoidal perturbation [6] (see Fig. 2(a)):

$$
\omega^{2}(t)= \begin{cases}\omega_{0}^{2} & \text { for } t \leq 0 \\ f(t)=\omega_{0}^{2}-\omega_{1}^{2}(1-\cos (2 \Omega t)) & \text { for } t>0\end{cases}
$$

The solution to Eq. (10) for $t>0$ is a Mathieu function:

$$
\eta(t)=A e^{\mu \Omega t} \sum_{n=-\infty}^{\infty} c_{2 n} e^{2 i n \Omega t}+B e^{-\mu \Omega t} \sum_{n=-\infty}^{\infty} c_{2 n} e^{-2 i n \Omega t}
$$

where $\mu$ and $\left\{c_{2 n}\right\}$ are determined by simultaneous equations (see in Ref. [4]), and $A$ and $B$ are complex constants chosen to satisfy $\eta(0)=1$ and $\dot{\eta(0)}=-i \omega_{0}$. Figure 3 shows $\mu$ as a function of $\omega_{0}, \omega_{1}$ and $\Omega$. The circle in Fig. 3 indicates the parameters for Fig. 2(a). In certain regions (white) of the parameter space $\mu$ is purely imaginary while in others (dark) it has a real part. This real part indicates that the classical particle is resonating with the oscillating dot; the particle's oscillations become infinitely large as $t \rightarrow \infty$. The corresponding effect on the quantum mechanical system, which depends on time only through $\eta(t)$, will be an increase in energy as $t \rightarrow \infty$ and a decrease in localization of $R(r, t)$ and hence $\psi_{\text {rel }}$. This spreading in $\psi_{\text {rel }}$ implies an increase in the average electron-electron separation, and will lead to electrons escaping from any realistic dot having a finite depth. For small $\omega_{1}, \mu$ has a real component only when the perturbing frequency $\Omega$ is equal to an integer fraction $\frac{1}{n}$ of the dot potential $\omega_{0}$. Note our treatment is exact for any amplitude $\omega_{1}^{2}$. The theory remains valid even when $\omega_{1}^{2}>\frac{1}{2} \omega_{0}^{2}$, in which case the dot becomes repulsive (i.e. an 'anti-dot') for part of each oscillation as shown in Fig 2(a). The region of Fig. 3 below the line $\bar{\omega}=\omega_{1}$ corresponds to such a system. Stable zones persist here, thus a dot may periodically become repulsive and yet confine its electrons for all time. Indeed in the limit $\omega_{1}=\omega_{0}$ (the bottom edge in Fig. 3) the $N$-electron droplet may remain localized for all time even though the time-averaged confinement potential is zero (i.e. $\bar{\omega}^{2}=\omega_{0}^{2}-\omega_{1}^{2}=0$ ). The sinusoidal perturbation in these white, stable zones generates an effective attractive interaction between electrons which competes with the electrostatic repulsion. At $\bar{\omega}=0$ the 
result is a stable, free-standing $N$-electron plasma droplet. From the inset in Fig. 3 we see that, for $\bar{\omega}=0$, the range of the parameter $\frac{\omega_{1}}{\Omega}$ over which the system is stable (i.e. white zones) is small but finite for larger $\frac{\omega_{1}}{\Omega}$; we note that there an infinite number of such stable zones which are distributed along the entire $\bar{\omega}=0$ axis.

Varying only $\Omega$ corresponds to moving along a radial line in Fig. 3. Consider the indicated line $\bar{\omega}=\omega_{1}$; moving out along this line switches the system between unstable states (dark zones - system absorbs energy from the perturbing field) and stable states (white zones - no net absorption). This pattern seems to persist arbitrarily far along this radial line, hence an experiment to study this stability effect could be performed at any frequency $\Omega$ which is convenient, irrespective of $\omega_{0}$. This observation is encouraging experimentally since the frequency $\omega_{0}$ is typically high $\left(10^{12} \mathrm{~Hz}\right.$ for $\left.\hbar \omega_{0}=1 \mathrm{meV}\right)$.

We now focus on 'stable' solutions (white zones in Fig. 3). Figure 2(b) shows the timevariation of the r.m.s. electron-electron separation $\bar{r}(t)$ for a sinusoidal perturbation (Fig. $2(\mathrm{a})$ ); it seems to show a chaotic irregularity (N.B. the amplitude is always finite since we are in the stable regime). The function $\bar{r}(t)$ is actually the square-root of a sum of cosine functions; Fig. 2(c) shows the amplitudes and frequencies of the dominant terms in this sum. They are made up of two distinct groups: one at frequencies $2 n \Omega$ and another at $(2 n+1) \Omega \pm \beta \Omega$. Two lines from the second group are labeled as an example. The value of $\beta$ may be found from Fig. 3; for the present choice of parameters (marked by a circle in Fig. 3) $\beta=2.35$. Figure 3 shows that when the perturbation amplitude $\omega_{1}$ is small then $\beta \approx \frac{\bar{\omega}}{\Omega}$. Thus for weak-to-moderate perturbations, the second group of peaks in the frequency spectrum lie at sums and differences of $\bar{\omega}$ and the driving frequency $\Omega$, i.e. at $(2 n+1) \Omega \pm \bar{\omega}$. The system can hence exhibit significant frequency mixing and harmonic generation. Figure 3 shows how $\beta$ (and hence the spectrum) changes as we move to the strongly non-linear regime $\left(\omega_{1} \approx \bar{\omega}\right)$. The parameters for Fig. 2 correspond to a strong perturbation $\left(\omega_{1}^{2}=0.57 \omega_{0}^{2}\right)$ which causes $\Omega \beta$ to deviate from $\bar{\omega}$ by $16 \%$.

In summary, we have presented a method for finding the exact dynamical response of an $N$ electron droplet. Our first example shows that a droplet subjected to a sudden increase in 
confinement will exhibit novel size oscillations associated with dynamical breathing modes for all time. Our second example shows that a sinusoidally varying confinement generates strongly non-linear size oscillations whose stability depends non-trivially on the confinement parameters. Electrons may remain localized even in the absence of a time-average confinement potential, thereby offering a possible new method of quantum dot fabrication. This plasma droplet could be moved around in the plane under the influence of a small, static in-plane electric field. In both examples the size oscillations are strong in that they involve all the electrons in the dot, and should therefore be readily observable experimentally.

We thank Luis Quiroga and Nikos Nicopoulos for useful discussions. S.C.B. is supported by an EPSRC studentship. 


\section{REFERENCES}

[1] For a review, see for example T. Chakraborty, Comment. Condensed Mat. Phys. 16, 35 (1992) or N.F. Johnson, J. Phys.: Condens. Matt. 7, 965 (1995).

[2] L.D. Hallam, J. Weis and P.A. Maksym, Phys. Rev. B 53, 1452 (1996).

[3] N.F. Johnson and L. Quiroga, Phys. Rev. Lett. 74, 4277 (1995).

[4] I.S. Gradshteyn and I.M. Ryzhik, Tables of Integrals, Series and Products (5 ${ }^{\text {th }}$ Edition, Academic Press, London, 1994), Eq. 8.975-1.

[5] P. Camiz, A. Gerardi, C. Marchiori, E. Presutti and E. Scacciatelli, J. Math. Phys. 12 2040, (1971).

[6] The B-field can be trivially introduced by replacing $\omega_{0}$ by $\omega_{0}(B)$. 


\section{Figure Captions}

Figure 1. (a) Step-like perturbation $\omega^{2}(t)$. (b) $\eta(t)$; inset shows the projection onto the complex $\eta$ plane. (c) The r.m.s. electron-electron separation $\bar{r}(\mathrm{t})$.

Figure 2. (a): Sinusoidal perturbation $\omega^{2}(t) ; \bar{\omega}^{2} \equiv \omega_{0}^{2}-\omega_{1}^{2}$. (b) The r.m.s. electron-electron separation $\bar{r}(\mathrm{t})$. (c) Frequency (Fourier) composition of $\bar{r}^{2}(t)$.

Figure 3. Top: Contour plot showing $\mu$ as a function of perturbation parameters; $\mu=$ $\operatorname{Re}\{\mu\}+i \beta$. White regions: $\operatorname{Re}\{\mu\}=0$ and contours are lines of constant $\beta$; on $\omega_{1}=0$ axis

$\beta=\frac{\bar{\omega}}{\Omega}$. Dark regions: $\operatorname{Re}\{\mu\}>0$ and contours are lines of constant $\operatorname{Re}\{\mu\}$ in increments of 0.2 , as shown by key. 
Fig. 1

a)

b)
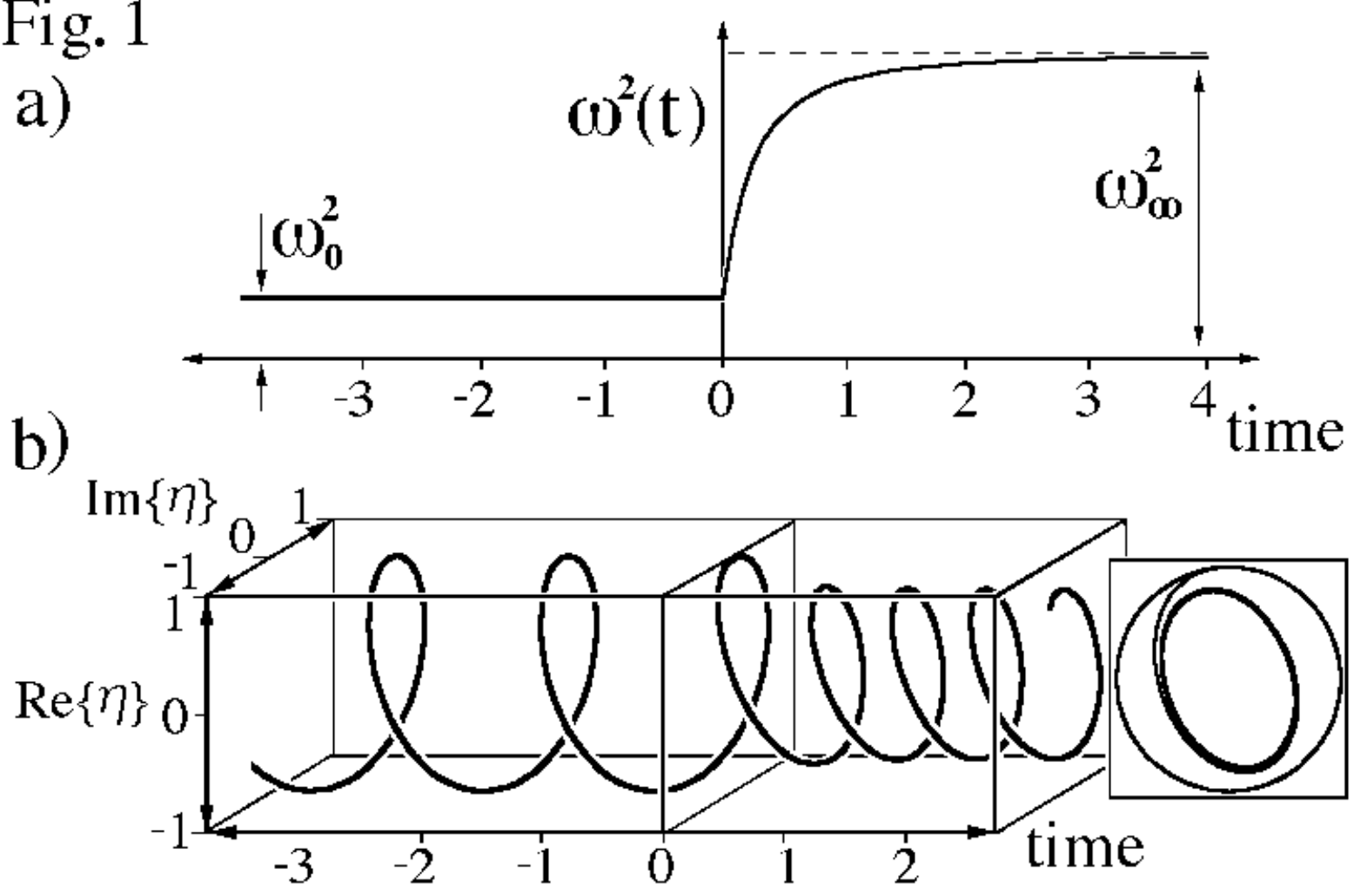

c)

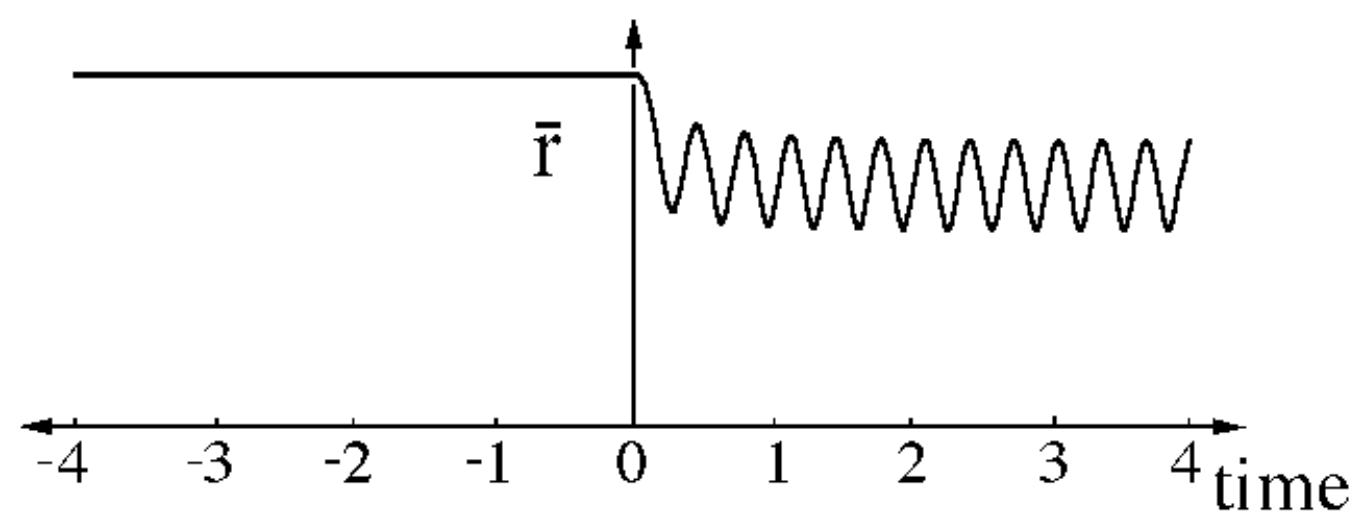


Fig. 2

a)

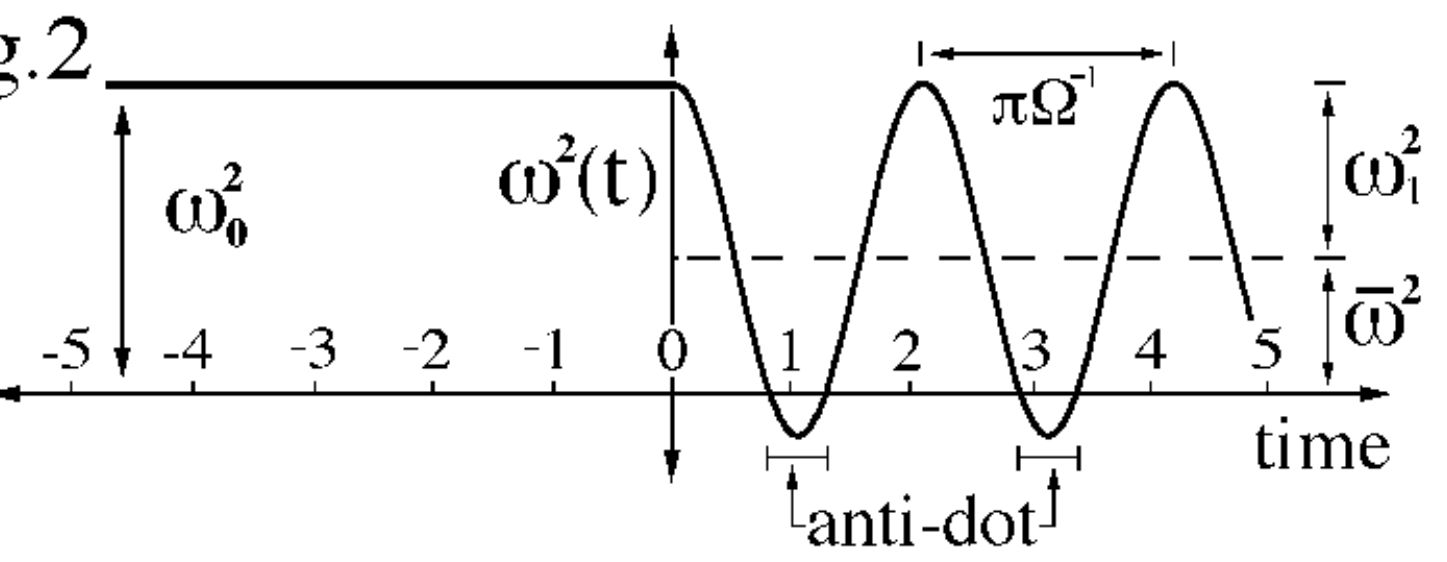

b)

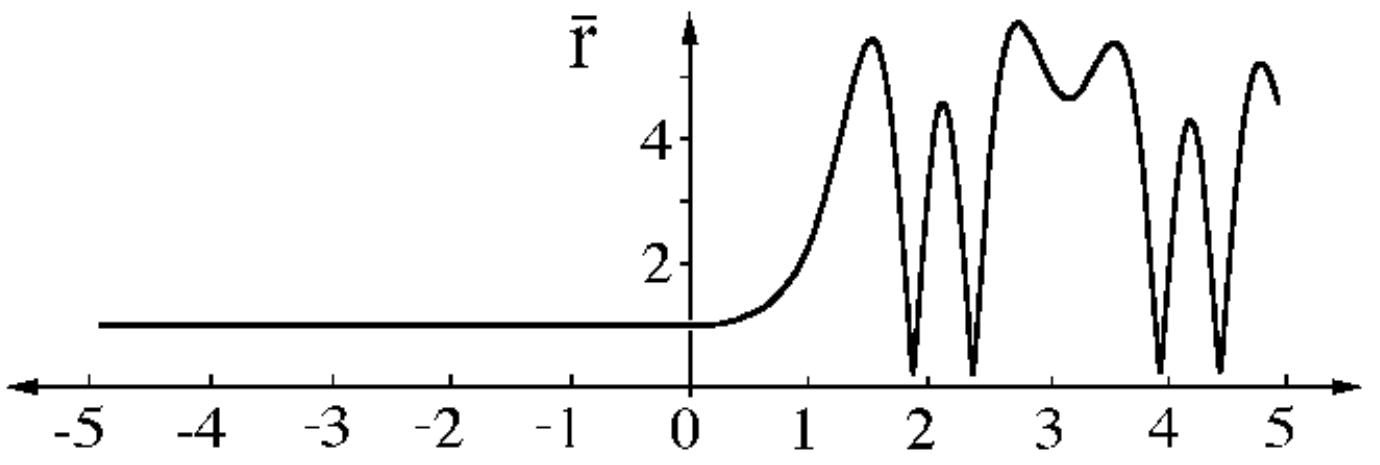

c)

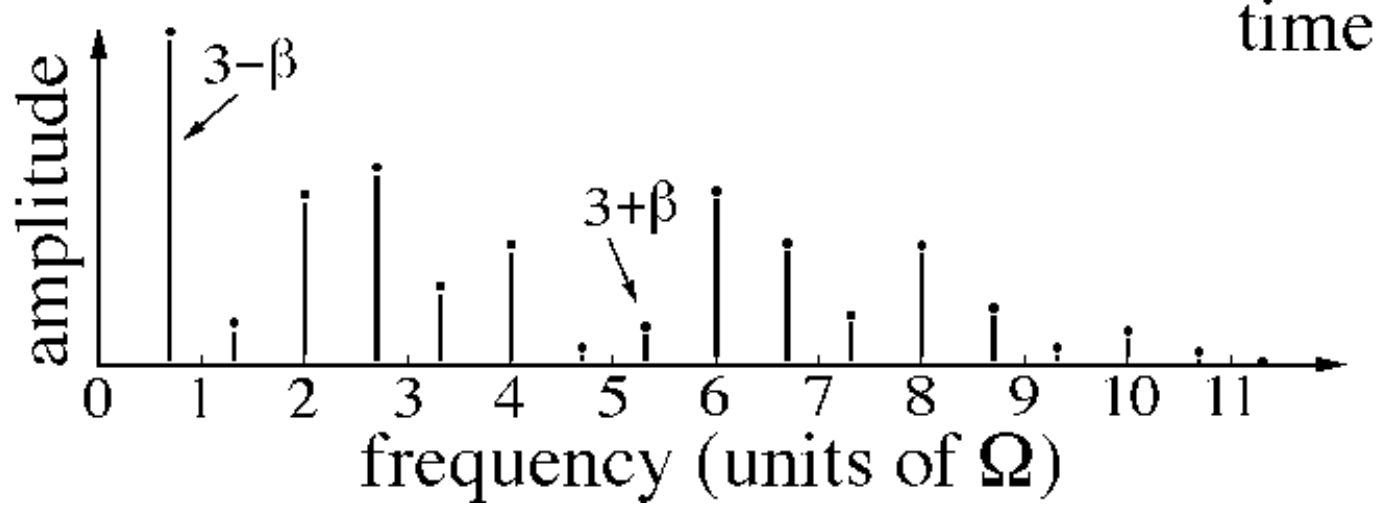




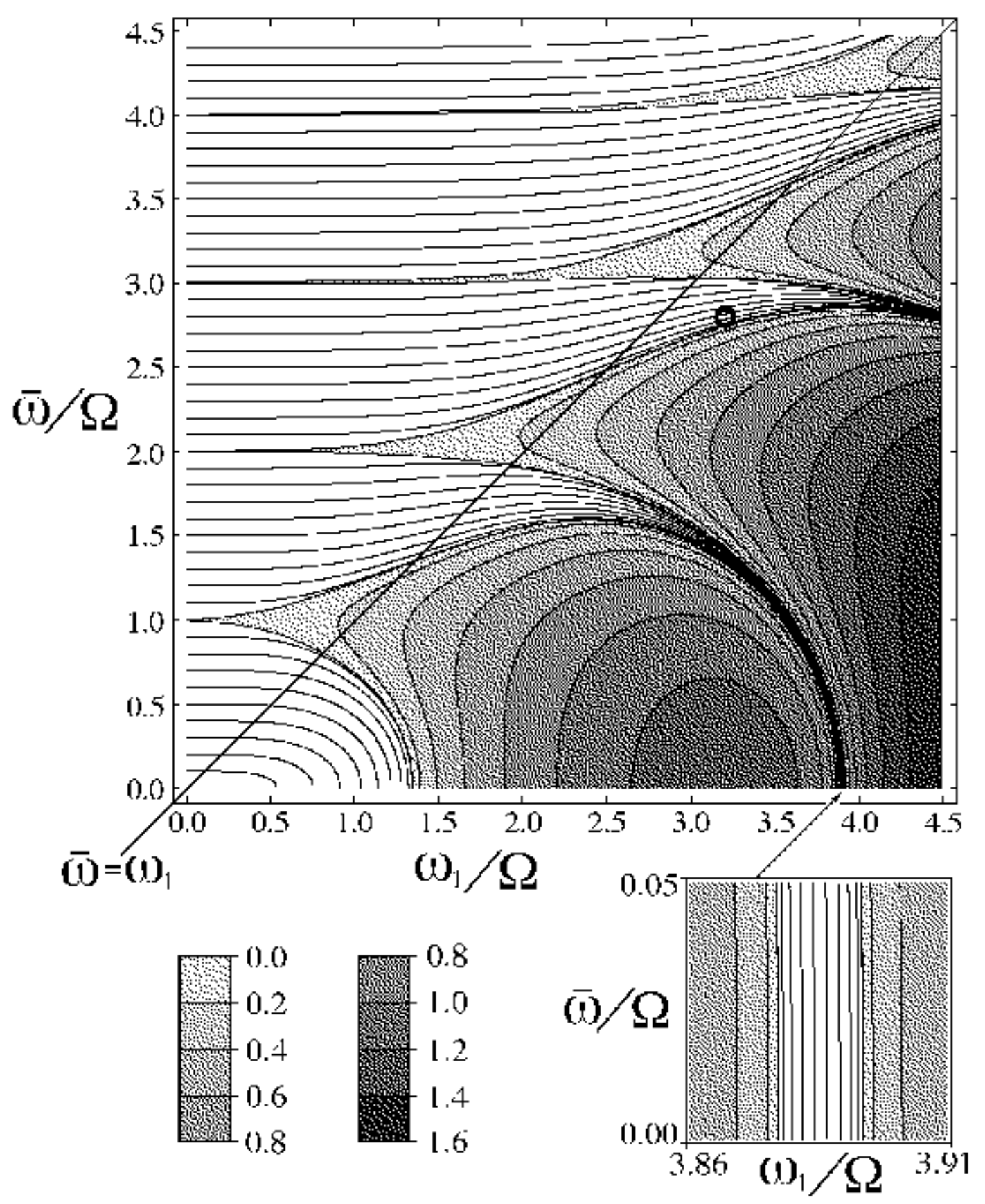

